\title{
The Nature of Compact Groups
}

\author{
H. M. Tovmassian
}

Instituto Nacional de Astrofísica Optica y Electrónica, AP 51 y 216, 72000, Puebla, Pue., México

H. Tiersch

Sternwarte Königsleiten, Munich, Germany

O. Martinez and O. Yam

Instituto Nacional de Astrofísica Optica y Electrónica, AP 51 y 216, 72000, Puebla, Pue., México

\begin{abstract}
We show that compact groups (CGs) are real physical entities, that almost all HCGs are dynamically associated with generally elongated loose groups of galaxies, that HCGs are compact cores of loose groups, and that they are longer living formations.
\end{abstract}

\section{Introduction}

According to N-body simulations, members of compact groups (CGs) should interact and merge to form a single elliptical galaxy on a time scale of a few orbital periods (Barnes 1989; Mamon 1986). For this reason the very existence of CGs was questioned. It was suggested that CGs are just chance alignments in loose groups (LGs) of galaxies (Mamon 1986, Walke \& Mamon 1989), or filaments seen end-on (Hernquist, Katz \& Weinberg 1995, Ostriker, Lubin \& Hernquist 1995).

We reconsidered the problem of the nature of CGs, and provide firm arguments in favor of their physical reality. We show also that CGs are cores of loose groups (LGs).

\section{CGs are real physical formations}

From the lists of Hickson Compact Groups (HCGs) and Shakhbazian compact groups (ShCGs) we separated two types of groups with extreme shapes: a chainlike (type I) and a round groups (type II), and compared their properties. In the case of HCGs we included the chain-like groups with a ratio $b / a<0.3^{1}$ into

\footnotetext{
${ }^{1} a$ is the angular distance between the most widely separated galaxies in the group, and $b$ is the sum of the angular distances $b_{1}$ and $b_{2}$ of the most distant galaxies, on either side of the line $a$ joining the most separated galaxies.
} 
type I groups. In the case of ShCGs, which have more member galaxies, we included into type I the groups with $b / a<0.4$. In both cases the groups with a round shape, for which $b / a>0.5$, form type II groups.

Oleak et al. (1998) and Malykh \& Orlov (1986) showed that ShCGs and HCGs have a prolate spheroidal configuration ("cigars"). If CGs are real physical entities, and have cigar-like configurations, then their member galaxies move in elongated orbits. The orientation of the most of chain-like groups is close to the perpendicular to the line of sight. As a consequence, the velocity dispersion of physical chain-like groups should, on average, be smaller than that of round groups, which are aligned almost along the line of sight. We find that this is just the case. The mean value of $\sigma_{v}$ for chain-like groups is equal to 194.3 , and is smaller than that of the round groups, $270.7 \mathrm{~km} \mathrm{~s}^{-1}$. Standard deviations are equal to \pm 119.2 and $\pm 130.5 \mathrm{~km} \mathrm{~s}^{-1}$ respectively. Kolmogorov-Smirnov (K-S), Student- $t$ and Mann-Whitney two-sample tests showed that the two distributions are significantly different. The difference between the mean values of $\sigma_{v}$ is statistically significant with a confidence level $>95 \%$.

Thus, data on radial velocity dispersion of HCGs show that HCGs are real physical formations. If CGs are a result of a chance projection of field galaxies, there should not be any difference in $\sigma_{v}$ for the two types of groups.

Round groups of type II have in the mean more members (4.6) than elongated groups of type I (3.9) and hence have larger masses. Consequently the velocities of individual galaxies in such groups must according to the virial theorem be larger, and thus they must have larger $\sigma_{v}$. Hence, one may suggest that the observed difference in $\sigma_{v}$ of elongated and round groups is caused by the difference in the number of group members.

A consideration of the average values of $\sigma_{v}$ of type I and type II groups consisting of 3,4 and 5 members shows, however, that in all samples the average $\sigma_{v}$ of round groups is larger. The Student- $t$, Mann-Whitney and the K-S tests showed that the differences in $\sigma_{v}$ between the groups of types I and II with 3,4 and 5 members respectively are not statistically significant. However, the combined probability of having by chance larger $\sigma_{v}$ for all three samples of round groups simultaneously, is equal to 0.04 . This is fairly significant. Thus, the suggestion that the dependence of $\sigma_{v}$ on $b / a$ is a result of a possible dependence of $\sigma_{v}$ on the number of group members is not valid.

The fact that $\sigma_{v}$ is on average greater for groups with more members, independently proves (from the virial theorem) that the groups considered are gravitationally bound systems, and hence real physical formations.

We give further arguments below in support of our conclusion on the reality of CGs.

- The mean projected linear widths $b$ of 44 chain-like groups is equal to $13.6 \pm 10.4 \mathrm{kpc}$, and is about 3.5 times smaller than the widths of 24 round groups. Conditions in very narrow physical chain-like groups are favorable for tidal interactions and merging. We found that 70 out of 171 galaxies are in chain-like groups, i.e. $41 \%$, show signs of interaction and/or merging, while in type II groups only 27 galaxies out of 110 , i.e. $24 \%$, show such signs. The relative number of groups with three or more interacting (I) and/or merging (M) members is $\sim 0.32$ in type $\mathrm{I}$, and is $\sim 0.17$ in 
type II. The data on I/M members are taken from Mendes de Oliveira \& Hickson (1994).

Thus, the relative number of galaxies with signs of interaction and/or merging in type I groups is, as predicted, two times larger than the number of interacting/merging galaxies in round groups.

The relative number of groups without I/M galaxies is, on the contrary, smaller in groups of type I: $\sim 23 \%$ in type I, and $\sim 29 \%$ in type II. The fraction of groups without $\mathrm{I} / \mathrm{M}$ galaxies is larger in groups with large velocity dispersion. 3 out of $12(25 \%)$ type I HCGs with $\sigma_{v}>250 \mathrm{~km} \mathrm{~s}^{-1}$ do not contain I/M members. This proportion is even larger in type II groups: 9 out of 13 groups $(69 \%)$. The larger proportion of groups without I/M galaxies among groups with larger $\sigma_{v}$, i.e. with higher velocities, is apparently due to the lower efficiency of interaction and/or merging processes.

The fraction of $\mathrm{E}$ and $\mathrm{S} 0$ galaxies in groups of both types that do not contain I/M members is $71 \%$. It is larger than for groups containing I/M members, $52 \%$. One may suggest that in groups without $\mathrm{I} / \mathrm{M}$ galaxies, the dynamical evolution is much more advanced. When a merging process is completed, we see a higher proportion of $\mathrm{E}$ and $\mathrm{S} 0$ galaxies.

Hence, the differences in fractions of $\mathrm{I} / \mathrm{M}$ and $\mathrm{E}, \mathrm{S} 0$ galaxies in groups of types I and II show that interaction and merging processes are, as we predicted, more efficient in elongated, chain-like groups.

- The difference in magnitudes between the brightest and the second brightest galaxy in the group, $m_{1}-m_{2}$, decreases systematically with the increase of the number of member galaxies in HCGs. For groups of both types with $3,4,5$, and 6 or 7 members this difference is equal to $1.05 \pm 0.7(20)$, $0.71 \pm 0.5(27), 0.61 \pm 0.5(15)$, and $0.4 \pm 0.2(5)$, respectively. The corresponding numbers of groups are shown in parenthesis. Magnitudes are taken from Hickson (1994). If several merging processes have already occurred in a group then the central (cannibal) galaxy becomes brighter, and the group would contain smaller number of members. The greater the number of galaxies in a group, the fewer mergings have occurred in it, and the smaller should be the difference in magnitude between the cannibal galaxy and the other galaxies in the group.

Hence, the differences in $m_{1}-m_{2}$ in groups with different number of members allow us to suggest that interaction and merging processes are actually going on in CGs. It follows that groups with smaller number of members are in more evolved stages.

- A consideration of the surface density distribution showed that the surface density is increasing towards the centers in both types of groups. The reason for the concentrated surface density distribution may be intrinsic.

- It cannot be excluded that some groups with very large $\sigma_{v}$ may not be real physical groups, but a result of optical projections. If we assume arbitrarily that groups with $\sigma_{v}$ exceeding $400 \mathrm{~km} \mathrm{~s}^{-1}$ result from chance projection, then at most 3 out of 44 groups of type I (about 7\%), and 4 out 
of 24 groups of type II (about 17\%) may be false optical groups. Hence, the number of false groups may be by a factor 2 more in round groups.

All of the above items allow us to conclude that CGs do not result from chance projection of field galaxies or filaments seen end-on, but are real physical formations.

\section{Compact groups are the cores of loose groups}

Rood \& Williams (1989) (hereafter RW) and Ramella et al. (1994) (hereafter RDGH) showed that some HCGs are associated with larger LGs. In order to "save" the existence of HCGs Governato et al. (1996) suggested that field galaxies from LGs fall from time to time onto CGs, and thus preserve their existence.

If galaxies of LGs are unrelated to CGs, then their distribution around CGs should be random. However, if we suppose that the LG galaxies are dynamically associated with CGs then we have to expect a certain correlation of the distribution of the supposed LG galaxies with the orientation of CG. We find out that this is indeed the case.

To see whether there is any correlation between the orientation of the CGs and the distribution of LG galaxies around them we used the RW and RDGH lists of LGs. RW looked for LGs in the area within a ten HCG radius. RW assumed that the surrounding area of a HCG contains an extra number of neighbors, if the ratio of signal to Poisson noise, $S / N=\left(N_{\text {observed }}-\right.$ $\left.N_{\text {field }}\right) /\left(N_{\text {field }}\right)^{1 / 2}>1$. For pure Poisson noise from superposition of field galaxies, $\mathrm{S} / \mathrm{N}$ has an average of 0 and a standard deviation of 1 . Groups with $S / N>1$ were considered as having statistically detected neighbors.

RDGH examined the relationship between HCGs and their neighborhoods in the area with a characteristic radius $1.5 h^{-1}$, and within the velocity range of $1500 \mathrm{~km} \mathrm{~s}^{-1}$ from the median radial velocity of the group. RDGH considered the presence of LG as certain, if $N_{n} \gg N_{i n t}$, where $N_{n}$ is the number of neighbors in redshift space, and $N_{i n t}$ is the number of expected interlopers.

Member galaxies in HCGs are generally moving in orbits oriented along the elongation of the group. If galaxies of the LG are dynamically associated with the CG then they should move in analogous but more elongated orbits with the same orientations. The number of LGs detected will depend on the orientation of the CG relative to the the line-of-sight; most LGs will be detected when the CG is aligned at intermediate angle between the line-of-sight and the celestial plane. At low angles to the line-of-sight, LGs will be projected onto the CG and might be considered proper members. At large angles to the line-of-sight the search radius of $10 \mathrm{CG}$ radii will preferentially exclude more distant LGs. Thus the maximal value of the relative number of detected LGs would be reached at values of $b / a$ somewhat less than 0.7 .

We show in Fig. $1 b / a$ versus relative numbers of HCGs associated with LGs, where we have used data on 88 accordant redshift HCGs from the RW, and 38 HCGs from the RDGH samples. In the latter case we considered the data related to the region of the complete CfA survey. For a smaller number of RDGH groups we considered the relative numbers of HCGs with associated LGs in bins twice as large as for the RW sample. 


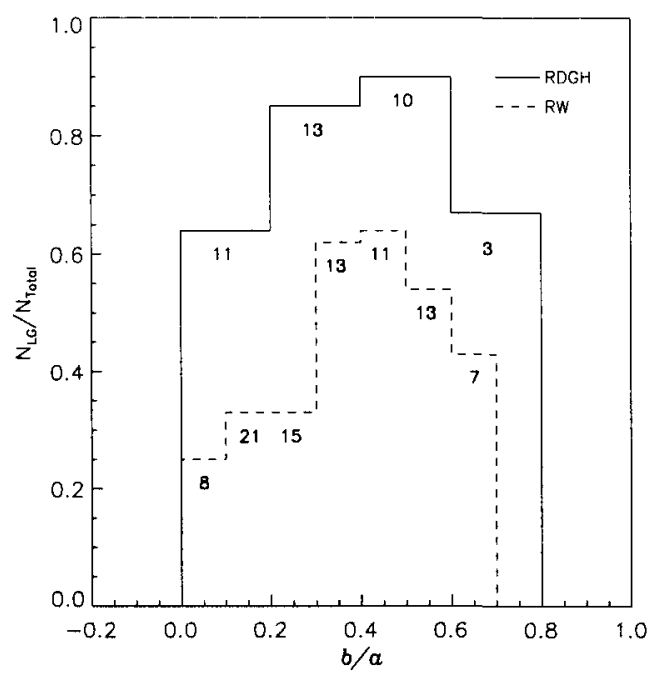

Figure 1. The dependence of the relative number of HCGs with associated loose groups on the ratio $b / a$. The total numbers of groups in each bin are shown.

Fig. 1 shows that for both samples the relative number of HCGs associated with LGs is indeed correlated with the elongation $b / a$ of groups. As we expected, the relative numbers of HCGs with associated LGs are small at small values of $b / a$, increase for median values of $b / a$, and decrease for higher values of $b / a$, i.e. the observed distribution is similar to a normal distribution.

The two-sample Mann-Whitney rank sum test shows that the RW and RDGH distributions do not differ from each other at the $<0.01$ significance level. On the other hand, the Kolmogorov-Smirnov goodness-of-fit test shows that the RW sample with the mean value $\mu=0.49$ and $\sigma=0.18$, and the RDGH sample with $\mu=0.45$ and $\sigma=0.21$, do not differ from a normal distribution at a significance level $<0.01$ for both samples.

The correlation found between the relative numbers of HCGs associated with LGs, and $b / a$ allows us to conclude that galaxies of LGs are physically associated with corresponding HCGs.

It is remarkable that LGs were found by RDGH in about $90 \%$ out of 23 HCGs at median values of $b / a$, where conditions for detection of associated LGs are favorable. Therefore, we may conclude that almost all HCGs have associated LGs. Many LGs were not found only because of selection effects imposed by the group orientation.

When the orientation of the CG approaches the line of sight, i.e. when it is observed as a round group, almost all galaxies of the LG are projected onto the group itself. If the LG has only a few members then in some cases we would still see a CG, but with a larger number of members. This does appear to be the case. However, if LGs have a larger number of members projected onto the 
CGs, then some CGs may not satisfy the compactness criterion, and would not be detected. Therefore, there should be a smaller number of round HCGs than the chain-like ones. As we saw, the number of round HCGs is, indeed about half the number the chain-like groups.

\section{Conclusions}

The compact group (CG) samples considered allow us to conclude that:

- CGs are real physical formations.

- Some of the galaxies found in the neighborhood of Hickson Compact Groups (HCGs) are not just field galaxies that fall from time to time onto the CGs, and thus maintain their existence. Rather, these galaxies are constituent members of CGs, and are gravitationally bound to them. Hence, CGs do not exist in isolation, and have members located at relatively large distances from them.

- HCGs are compact cores of larger, elongated systems - the Loose Groups (LGs).

- Since HCGs have a certain amount of distant members, they are probably more stable and longer living systems than has been generally assumed.

Acknowledgments. HMT is grateful to the IAU for a travel grant, and the LOC for paying the registration fee.

\section{References}

Barnes J. 1989, Nature, 338, 123

Governato F., Tozzy P., Cavaliere A. 1996, ApJ, 458, 18

Hernquist L., Katz N., Weinberg D. H. 1995, ApJ, 442, 57

Hickson P. 1994, Atlas of Compact Groups of Galaxies, Gordon and Breach, New York

Malykh S. A., Orlov V. V. 1986, Afz, 24, 445

Mamon G. A. 1986, ApJ, 307, 426

Mendes de Oliveira C., Hickson P. 1994, ApJ, 427, 684

Oleak H., Stoll D., Tiersch H., MacGillivray H. T. 1998, Astron. Nachr., 319, 235

Ostriker J. P., Lubin L. M. \& Hernquist L. 1995, ApJ, 444, L61

Ramella M., Diaferio A., Geller M. J., Huchra J. P. 1994, AJ, 107, 1623

Rood H. J. \& Williams B. A. 1989, ApJ, 339, 772

Walke D. G., Mamon G. A. 1989, A\&A, 225, 291 